\title{
Neutrophil-Lymphocyte Ratio as a Reliable Predictive Marker in Systemic Lupus Erythematosus
}

\author{
HEBATU ALLAH A. EL-HABIBY, M.Sc.*; NOHA E. ESHEBA, M.D.*; ENAAM S. ABD EL-BAR, M.D.** and \\ GEHAN M. SHEIRA, M.D.*
}

The Departments of Internal Medicine* and Clinical Pathology**, Faculty of Medicine, Tanta University

\begin{abstract}
Background: SLE is a complex autoimmune disease with heterogenous clinical manifestations and disease course, Nephritis is a major cause of morbidity and mortality in patients with lupus. Many clinical parameters and laboratory markers can be used to evaluate disease activity. NLR is positively associated with inflammatory disorders.

Objectives: Is to evaluate the Neutrophil Lymphocytic ratio (NLR) as a reliable predictive and prognostic marker in systemic lupus erythematosus.

Methods: The present study was carried out on 60 patients and 20 healthy individuals as controls. Patients were classified into: Group I: 40 SLE patients with active disease, which was sub divided into two subgroups: Group IA included thirty SLE patients with LN and Group IB included ten SLE patients with active disease without nephritis. Group II: 20 SLE patients with inactive disease. Group III: 20 apparently healthy volunteers as controls. CBC, serum creatinine, ESR, CRP, ANA, Anti-ds DNA, C3, C4, 24-hour protein in urine and urine analysis were done to all participants.

Results: The NLR of SLE patients was significantly higher compared to that of the controls. Furthermore, SLE patients with nephritis had higher NLR levels than those without nephritis.

Conclusion: NLR is a useful, simple and bed side inflammatory marker for assessment of disease activity in patients with SLE. Also, NLR is a promising predictor of lupus nephritis.
\end{abstract}

Key Words: Neutrophil Lymphocytic Ratio (NLR) - Systemic lupus erythematosus - Lupus nephritis.

\section{Introduction}

SLE is a complex autoimmune disease with heterogenous clinical features and disease course, characterized by pathogenic autoantibody formation, immune complex deposition, and end organ damage [1].

Correspondence to: Dr. Hebatu Allah A. El-Habiby, The Department of Internal Medicine, Faculty of Medicine, Tanta University
SLE patients continue to have disease activity 10 years after diagnosis even with appropriate management [2]. The disease activity appears to be an important predictor of both mortality and organ damage [3]

Renal affection may be the first symptomatic finding in SLE patients. It often occurs within one year of diagnosis but sometimes within the first five years after diagnosis [4].

Nephritis is a major cause of morbidity and mortality in patients with lupus [5]. Many patients do not respond even to aggressive therapy and progress to End Stage Renal Disease (ESRD) [6] .

Evaluation of disease activity with simple, available and low costly marker with high sensitivity is a major challenge [7].

Many studies have shown that NLR is positively associated with inflammatory disorders [8-10]. The use of Neutrophil-Lymphocyte Ratio (NLR) to evaluate the inflammatory response in SLE has not been established yet and in need for further studies.

\section{Patients and Methods}

This study was conducted on 60 patients with SLE admitted to inpatient wards and Outpatient clinic of Internal Medicine Department of Tanta University Hospital between January 2016 and June 2016 and 20 healthy age and gender matched volunteers were enrolled as the control group. All participant provided informed written consent and the study was approved by Tanta Faculty of Medicine Ethical Committee. 
The participants were divided into the following groups:

1- Group (1): 40 SLE patients with active disease, who were sub divided into:

- Group 1A: 30 with lupus nephritis.

- Group 1B: 10 without nephritis.

2- Group (2): 20 SLE patients with inactive disease (remission).

3- Group (3): 20 apparently healthy individuals as a control group matched age and sex.

Inclusion criteria:

Patients with 4 or more out of the 11 revised classification criteria for SLE of the American College of Rheumatology (ACR) were included in the study [11]. All patients were naive. Patients with SLEDAI > 8 were considered to have active disease [12]

\section{Exclusion criteria:}

Patients with active infection (all subjects in this study had negative CRP level), malignancies, acute poisoning, thrombus formation and ischemic injury, myocardial infarction, heart failure, diabetic nephropathy and other autoimmune diseases.

All participants in this study were subjected to: Thorough history taking, full clinical examination, laboratory investigations in the form of: Full blood count, blood urea, serum creatinine, fasting and $2 \mathrm{hr}$ post prandial blood glucose, Erythrocyte Sedimentation Rate (ESR), C-Reactive Protein (CRP), Anti-Nuclear Antibody (ANA), anti-dsDNA, serum complement 3 and 4 (C3 \& C4), Urine Analysis (UA), 24hr. Proteinuria, pelvi-abdominal ultra sound, renal biopsy in indicated cases for histopathological examination for classification of nephritis.

\section{Sampling and laboratory investigations:}

Sampling and all laboratory investigations were done in Clinical Pathology Department, Tanta University Hospitals.

Full blood count: Was performed using BCC3000 Auto Hematology Analyzer.

Anti-Nuclear Antibody (ANA): Was performed using a substrate containing human nuclei, such as HEp- 2 cells.

Anti-ds DNA: Was done by ELISA supplied by Calbiotech, catalog NO. DD037G.
Serum complement levels (C3\& C4): C3 was done by turbidimetry, supplied by BioSystems; catalog No COD 31084.

C4 was done by turbidimetry, supplied by BioSystems; catalog No COD 31085.

Statistical analysis of the data:

Data were fed to the computer and analyzed using IBM SPSS software package Version 20.0. Quantitative data were described using range (minimum and maximum), mean, standard deviation and median. Comparisons between more than two populations were analyzed using F-test (ANOVA). ROC-curve was used for assessment of sensitivity and specificity. Significance of the obtained results was judged at the $p$-value $<0.05$.

\section{Results}

Comparison between the studied groups showed statistical significance as regard to hemoglobin, serum creatinine, $24 \mathrm{hr}$. urinary protein, ESR, ANA, Anti-ds DNA, C3 and NLR. In contrary comparison showed no statistical significance as regard age, sex, platelet count, $\mathrm{C} 4$ as shown in (Table 1).

As for NLR, it was significantly higher in Group IA in comparison to the other three groups. Also, it was significantly higher in Group IB in comparison to Groups II and III as shown in (Table 2).

In this study NLR showed positive correlation with statistical significance in Group IA with the following parameters: SLEDAI, WBCs and neutrophil count ( $p=0.001)$ for all. As for Group IB NLR showed positive correlation with statistical significance with the following parameters: SLEDAI and 24hr. urinary protein. While in Group II NLR showed positive correlation with statistical significance with neutrophil count. C3 showed negative correlation with statistical significance in Group II. As for the class of nephritis, there was no significant correlation between it and NLR in Group IA ( $p=0.979$ ) as shown in (Table 3).

As regard to renal biopsy, 26 patients out of the 30 who had lupus nephritis agreed to undergo renal biopsy the majority of the patients had class III and IV nephritis (38.5\% for each class) while $23 \%$ had class II nephritis as shown in Fig. (1).

The ROC curve showed that (considering a cutoff value of $1.5 \%$ for NLR): The sensitivity of NLR as a marker of SLE activity was $97 \%$, while it's specificity was $70 \%$ with $88 \%$ positive predictive value and $86 \%$ accuracy as shown in (Table 4) and Fig. (2). 
Table (1): Comparison between the studied groups.

\begin{tabular}{llllll}
\hline Parameter & $\begin{array}{c}\text { Group IA } \\
(\mathrm{n}=30)\end{array}$ & $\begin{array}{c}\text { Group IB } \\
(\mathrm{n}=10)\end{array}$ & $\begin{array}{c}\text { Group II } \\
(\mathrm{n}=20)\end{array}$ & $\begin{array}{c}\text { Group III } \\
(\mathrm{n}=20)\end{array}$ \\
\hline Age in years & $29.50 \pm 9.39$ & $26.10 \pm 8.91$ & $28.90 \pm 8.66$ & $28.75 \pm 6.90$ & $p$ \\
Sex (female/male) & $29 / 1$ & $9 / 1$ & $20 / 0$ & $19 / 1$ & 0.756 \\
Hb gm/dl & $8.71 \pm 2.05$ & $9.83 \pm 1.8$ & $10.13 \pm 2.01$ & $11.97 \pm 0.80$ & 0.580 \\
Platlet X 10 ${ }^{3} \mathrm{cell} / \mathrm{mm}^{3}$ & $207000 \pm 120233.7$ & $232600 \pm 146133.3$ & $213650 \pm 88388.66$ & $221200 \pm 48180.14$ & 0.908 \\
WBCs X 10 ${ }^{3} \mathrm{cell} / \mathrm{mm}^{3}$ & $6093.43 \pm 4252.27$ & $6458.50 \pm 3734.22$ & $5420.00 \pm 2371.90$ & $6141.00 \pm 1397.87$ & 0.824 \\
Serum Creatinine $\mathrm{mg} / \mathrm{dl}$ & $2.04 \pm 1.52$ & $0.84 \pm 0.23$ & $0.88 \pm 0.31$ & $0.81 \pm 0.22$ & 0.001 \\
24hr. ptn & $1954.47 \pm 1168.99$ & $277.40 \pm 107.49$ & $160.10 \pm 126.57$ & $170.00 \pm 52.39$ & 0.001 \\
ESR 1 & $72.90 \pm 34.06$ & $65.80 \pm 28.14$ & $56.94 \pm 37.27$ & $20.05 \pm 5.69$ & 0.001 \\
ANA & $10.14 \pm 17.78$ & $4.59 \pm 2.52$ & $18.96 \pm 23.63$ & $0.81 \pm 0.28$ & 0.006 \\
Anti-ds DNA & $351.17 \pm 385.83$ & $219.51 \pm 184.97$ & $189.19 \pm 236.06$ & $15.50 \pm 7.72$ & 0.001 \\
C3 & $56.79 \pm 60.20$ & $81.20 \pm 22.44$ & $112.45 \pm 16.98$ & $100.55 \pm 17.88$ & 0.001 \\
C4 & $14.77 \pm 20.02$ & $13.70 \pm 10.21$ & $19.81 \pm 6.95$ & $23.55 \pm 7.57$ & 0.113 \\
NLR & $5.07 \pm 2.57$ & $3.61 \pm 2.88$ & $2.02 \pm 0.84$ & $1.23 \pm 0.38$ & 0.001 \\
SLEDI & $23.30 \pm 11.99$ & $15.50 \pm 7.69$ & $5.50 \pm 1.19$ & $\mathrm{~N}$. A & 0.001 \\
\hline
\end{tabular}

Table (2): Comparison between the studied groups as regard to NLR.

\begin{tabular}{lllllll}
\hline & Range & Mean \pm S.D & F-test & $p$-value & \multicolumn{2}{c}{ Post Hock test } \\
\hline$N L R:$ & & & & & & \\
G IA & $1.95-12$ & $5.07 \pm 2.57$ & 18.916 & $0.001 *$ & $p 10.042^{*}$ & $p 40.036^{*}$ \\
G IB & $0.8-11.25$ & $3.61 \pm 2.88$ & & & $p 20.016^{*}$ & $p 50.002^{*}$ \\
G II & $0.7-3.9$ & $2.02 \pm 0.84$ & & & $p 30.001^{*}$ & $p 60.203$ \\
G III & $0.44-2.1$ & $1.23 \pm 0.38$ & & & & \\
\hline
\end{tabular}

p1: Group IA \& IB

p2: Group IA \& II.

p3: Group IA \& III.

p4: Group IB \& II

p5: Group IB \& III.

$p 6$ : Group II \& III.

Table (3): Correlations of NLR with other variables of the studied groups.

\begin{tabular}{lllcccc}
\hline \multirow{2}{*}{ With } & \multicolumn{6}{c}{ NLR } \\
\cline { 2 - 7 } & \multicolumn{2}{c}{ GIA } & \multicolumn{2}{c}{ GIB } & \multicolumn{2}{c}{ GII } \\
\cline { 2 - 7 } & $r$ & $p$ & & & & \\
\cline { 2 - 7 } SELDAI & 0.834 & $0.001^{*}$ & 0.887 & $0.001^{*}$ & 0.106 & 0.658 \\
Age & -0.184 & 0.329 & -0.481 & 0.159 & -0.259 & 0.271 \\
HB & -0.233 & 0.216 & 0.420 & 0.226 & -0.344 & 0.137 \\
PLT & 0.113 & 0.552 & -0.464 & 0.177 & 0.107 & 0.652 \\
WBC & 0.569 & $0.001^{*}$ & -0.020 & 0.969 & 0.182 & 0.443 \\
Neutrophil & 0.628 & $0.001^{*}$ & 0.141 & 0.697 & 0.481 & $0.032^{*}$ \\
Lymphocytes & -0.025 & 0.894 & -0.401 & 0.251 & -0.348 & 0.284 \\
ESR 1 & 0.099 & 0.609 & -0.365 & 0.299 & -0.028 & 0.911 \\
ANA & 0.260 & 0.164 & -0.456 & 0.186 & 0.161 & 0.498 \\
Anti-ds DNA & -0.036 & 0.850 & 0.078 & 0.830 & 0.138 & 0.32561 \\
C3 & -0.057 & 0.618 & 0.296 & 0.407 & -0.479 & $0.032^{*}$ \\
C4 & -0.173 & 0.369 & -0.044 & 0.904 & -0.120 & 0.615 \\
Scr & -0.021 & 0.915 & 0.043 & 0.906 & 0.359 & 0.120 \\
24hr. ptn & 0.105 & 0.581 & 0.668 & $0.035^{*}$ & 0.393 & 0.086 \\
Renal biopsy & 0.005 & 0.979 & Not applicable & Not applicable \\
\hline
\end{tabular}

Table (4): Sensitivity, specificity, positive prediction, negative prediction and accuracy of NLR as a marker of activity of SLE.

\begin{tabular}{rcccccc}
\hline AUC & Cutoff & Sensitivity & Specificity & PPV & \multicolumn{2}{c}{ NPV Accuracy } \\
\hline NLR $\mathbf{0 . 9 2 2}$ & 1.5 & 93 & 70 & 88 & 80 & 86 \\
\hline
\end{tabular}




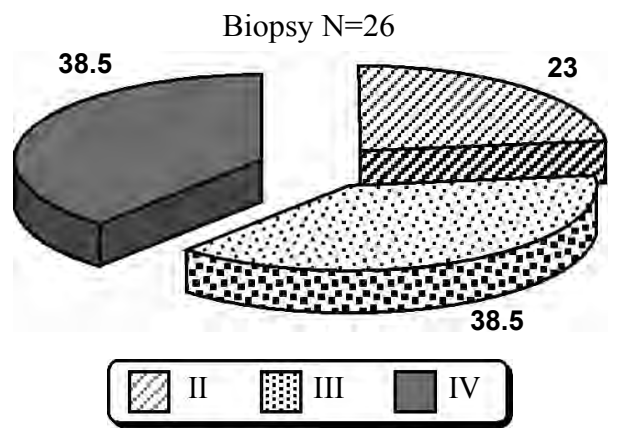

Fig. (1): Results of renal biopsy for patients of Group IA.

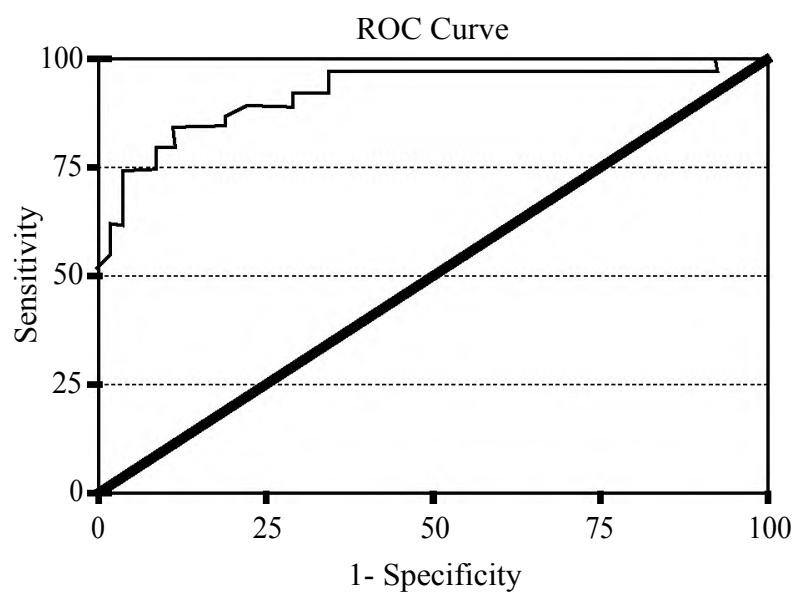

Fig. (2): Area under ROC curve denoting sensitivity of NLR for expecting activity of SLE.

\section{Discussion}

Lupus is a syndrome that primarily affects young women, its phenotypic variability makes every lupus patient unique with different clinical and laboratory characteristics [13].

Lupus Nephritis (LN) is one of the most common secondary glomerulonephritises. It is also a common and potentially devastating manifestation of lupus that occurs in more than half of SLE patients [14].

SLE disease activity is assessed via composite indices. However, routine clinical use of these indices is limited due to their impracticality [15] Therefore; non-invasive, simple, available and sensitive biomarker seems to be necessary.

Neutrophils and lymphocytes play major roles in inflammatory processes. Under inflammatory conditions, neutrophil and lymphocyte counts undergo temporary changes [16]

$\mathrm{CBC}$ is a basic investigation done in all admitted patients [17], The Neutrophil-Lymphocyte Ratio (NLR), calculated by dividing the ANC by the
ALC, can serve as an index of systemic inflammatory response in critically ill patients [18]

In this study NLR and its correlation to disease activity in 60 Egyptian patients with SLE; Group IA: 30 SLE patients with lupus nephritis, Group IB: 10 SLE patients with disease activity but without lupus nephritis (SLEDAI >_8) and Group II: 20 patients with inactive disease (SLEDAI $<8$ ) were investigated and compared to NLR in 20 healthy volunteers (Group III). All patients were diagnosed during their first hospitalization.

This study showed that there was no significant difference between the studied groups as regard age $(p=0.756)$ or gender $(p$-value $=0.580)$ and this is in agreement with Qin et al., [19], and with $\mathrm{Li}$ et al., [20]

As regards to $\mathrm{CBC}$, this study found that hemoglobin level was lower in group IA SLE patients with nephritis than group IB, group II and group III with statistical significance $(p=0.001)$. this was in agreement with Delgado-García et al., [21] and Yolbas et al., [22].

Mean platelet counts showed no statistical significance between the studied groups in agreement with Ayna et al., (2017) [23] and DelgadoGarcía et al., [21] . but in contrast to Qin et al., (2015) [19] who declared that platelet count was statistically decreased in SLE patients.

This study showed no significant difference between the studied groups as regard WBC ( $p=$ 0.824). This was in agreement with Delgado-García et al., [21].

Neutrophil count showed higher values in Group IA than Group IB, Group II showed lower levels than patients with active disease while neutrophil levels of Group III were the lowest with statistical significance $(p=0.046)$, these results were in contrast to Oehadian et al., [24] who showed no difference in neutrophil count between the groups, but the results of this work were in agreement with Qin et al., [19] who showed higher neutrophil counts in SLE patients and Ayna et al., [23] who stated that neutrophil counts were significantly lower in patients without nephritis.

The lymphocyte counts were significantly lower in SLE patients than controls and the lowest counts were those of Group IA followed by Group IB and Group II consecutively with statistical significance reflecting the inflammatory status of the disease. This comes in agreement with Oehadian et al., [24], Qin et al., [19] and Delgado-García et al., [21] . 
As regard to serum creatinine (S.Cr), this study showed higher values in patients with $\mathrm{LN}$ than other patients $(p$-value $=0.01)$ this was in agreement with Umare et al., [25] who found significant increase in creatinine levels in patients with active disease when compared to those with inactive disease $(p=0.192)$ and in contrast to DelgadoGarcía et al., [21] who showed no significant difference between groups $(p=0.6)$.

Mean 24-hour protein excretion in urine in Group IA LN patients was significantly higher in comparison to other groups and this was in agreement with El-Sayed et al., [26]

ESR was significantly higher in SLE patients especially those with nephritis. Wu et al., [27] showed the same results for ESR while Ayna et al., [23] determined that ESR was similar between groups.

ANA showed significant differences between active and inactive group of patients $(p=0.024)$ and $(p=0.006)$ for all groups, on the other hand Luo et al., (2017) [28] stated that there was no difference between active and inactive patients as regard ANA.

As regard, anti-ds DNA this study showed higher values in patients with LN than other groups, also showed higher values in patients with active disease without nephritis than inactive and controls ( $p=0.001)$, these results were similar to that reported by Yavuz et al., [29] and Luo et al., [28]

C3 showed lower levels in active patients than in active patients, also lower values were observed in patients with $\mathrm{LN}$ than patients without $\mathrm{LN}$, these results come in agreement with Yavuz et al., [29] and Luo et al., [28] who stated that C3 differed significantly between active and inactive patients. On the other hand, Elwy et al., [30] observed that levels of complement $\mathrm{C} 3$ and $\mathrm{C} 4$, did not differ between active and inactive disease, also they found that the clinical measurement of complement components is not sensitive enough to detect disease activity in SLE.

C4 showed no significant difference between the studied groups $(p=0.113)$ this result was consistent with Elwy et al., [30] and Luo et al., (2017) [28] but in the contrary Yavuz et al., (2014) [29] observed lower values of $\mathrm{C} 4$ in patients with active disease than other groups.

As regard to SLEDAI score there was a significant difference between the patients' groups, Luo et al., [28] also showed significant difference between active and inactive patients.

This study recorded higher levels of NLR in SLE patients with nephritis $(5.07 \pm 2.57)$ than SLE patients with active disease and without nephritis $(3.61 \pm 2.88)$, both groups showed higher levels than patients with inactive disease $(2.02 \pm 0.84)$ and the control group showed the least levels $(1.23 \pm$ 0.38 ). It also showed that, based on receiver operating characteristic curve (ROC curve) with area under the curve of 0.922 , cutoff NLR value of 1.5 , NLR had $93 \%$ sensitivity and $70 \%$ specificity in differentiating SLE patients with active disease with or without nephritis and patients with inactive disease.

These results were in agreement with Li et al., [20] who suggested that NLR can predict LN with a cutoff value of 4.4 for NLR (sensitivity 0.64 , specificity 0.91 ).

According to Oehadian et al., [24] cutoff NLR value of $\geq 1.93$ had sensitivity of $70 \%$ and specificity of $67 \%$ in differentiating SLE patients and normal subjects.

Qin et al., [19] used a cutoff value of 2.66 and reported $74.4 \%$ sensitivity and $77.5 \%$ specificity for prediction of SLE nephritis. While Ayna et al., [23] showed that cutoff NLR value of 1.93 had $83 \%$ sensitivity and $54 \%$ specificity in differentiating SLE patients with or without nephritis.

These results were in contrast to Yolbas et al., [22] who stated that there was no significant difference in NLR between the active and inactive SLE subgroups.

In patients with SLE with active disease, NLR correlated positively and significantly with SLEDAI scores $(r=0.834, p=0.01)$ for patients with nephritis and $(r=0.887, p=0.01)$ for patients without nephritis. This was in agreement with Wu et al., (2016) [27] and Qin et al., [19].

While the correlations of NLR with anti-ds DNA, C3 and C4 were not statistically significant in all groups. This was in agreement with Qin et al., [19] while Yolbas et al., reported that NLR was higher in hypo-complementemic SLE patients than in normo-complementemic patients [22].

In this study, NLR showed no obvious correlation with class of renal biopsy results. Ayna et al., [23]did not mention any correlation with biopsy, however their renal biopsy results were $(60.52 \%)$ proliferative LN (28.94\%) class V LN, (7.89\%) class VI LN, and (2.63\%) class II LN which were 
not consistent with our results; class III and IV nephritis ( $38.5 \%$ for each class) while $23 \%$ had class II nephritis, this may be due to different sample size. However, Delgado-García et al., (2016) [21] showed that $40 \%$ had ISN/RPS class III LN, $30 \%$ had class IV LN, $20 \%$ had class V LN, and $10 \%$ had class II LN which was near to our results.

\section{Limitations of the study:}

- It was a single center study with a cross-sectional design which limited the ability to infer a causal relation between NLR and disease activity.

- The study was based on a single measurement of WBC count that may not reflect the relation over time.

- The sample size was relatively small.

Conclusion:

- NLR was higher in SLE patients than controls.

- Higher ratios were observed during disease activity.

- NLR was more apparent in patients with lupus nephritis.

- NLR has a 93\% sensitivity in SLE as shown in this study.

So, NLR can be used as a possible simple bed side marker for SLE activity and prognosis specially with lupus nephritis.

\section{Recommendations:}

- More studies are needed on larger number of patients to focus on NLR and its relation with other markers of activity in SLE with or without nephritis for more precise elucidation and clinical usage of this marker.

- Future researches for assessing the role of NLR in other autoimmune disease should be put in mind.

- Restoring NLR to its normal value might be a potential therapeutic target in treating active SLE.

\section{Conflicts of interest:}

The authors declare no conflicts of interest.

\section{References}

1- ANOLIK J.H.: B cell biology and dysfunction in SLE. Bull NYU Hosp. Jt. Dis., 65 (3): 182-6, 2007.

2- PETRI M.: Hopkins Lupus Cohort. 1999 update. Rheum Dis Clin. North. Am., 26 (2): 199-213, 2000.

3- NOSSENT J.C.: SLICC/ACR Damage Index in AfroCaribbean patients with systemic lupus erythematosus: Changes in and relationship to disease activity, corticos- teroid therapy, and prognosis. J. Rheumatol., 25 (4): 654 $9,1998$.

4- BORCHERS A.T., LEIBUSHOR N., NAGUWA S.M., et al.: Lupus nephritis. A critical review. Autoimmun. Rev., 12 (2): 174-94, 2012.

5- AUSTIN H.A., BOUMPAS D.T., VAUGHAN E.M., et al.: High-risk features of lupus nephritis: Importance of race and clinical and histological factors in 166 patients. Nephrol. Dial. Transplant, 10 (9): 1620-28, 1995.

6- HSIEH C., CHANG A., BRANDT D., et al.: Tubulointerstitial Inflammation and Scarring Predict Outcome in Lupus Nephritis. Arthritis. Care Res. (Hoboken), 63 (6): 865-74, 2011

7- ILLEI G.G., GABOR G., TACKEY E., et al.: Biomarkers in systemic lupus erythematosus: II. Markers of disease activity. Arthritis \& Rheumatism, 50 (7): 2048-65, 2004.

8- LI M.X., LIU X.M., ZHANG X.F., et al.: Prognostic role of neutrophil-to-lymphocyte ratio in colorectal cancer: A systematic review and meta-analysis. Int. J. Cancer., 134 (10): 2403-13, 2014.

9- TAMHANE U.U., ANEJA S., MONTGOMERY D., et al.: Association between admission neutrophil to lymphocyte ratio and outcomes in patients with acute coronary syndrome. Am. J. Cardiol., 102 (6): 653-7, 2008.

10- HUANG W., HUANG J., LIU Q., et al.: Neutrophillymphocyte ratio is a reliable predictive marker for earlystage diabetic nephropathy. Clin. Endocrinol., 82 (2): 229-33, 2015

11- HOCHBERG M.C.: Updating the American College of Rheumatology revised criteria for the classification of systemic lupus erythematosus. Arthritis. Rheum., 40 (9): 1725, 1997.

12- FREIRE E.A.M., SOUTO L.M. and CICONELLI R.M.: Assessment measures in systemic lupus erythematosus. Revista brasileira de reumatologia, 51 (1): 75-80, 2011.

13- SAEED M.: Lupus pathobiology based on genomics. Immunogenetics, 1-12, 2017.

14- KATEWA A., WANG Y., HACKNEY J.A., et al.: Btkspecific inhibition blocks pathogenic plasma cell signatures and myeloid cell-associated damage in IFNa-driven lupus nephritis. JCI insight, 2 (7), 2017.

15- LIU C.C. and AHEARN J.M.: The search for lupus biomarkers. Liu CC, Ahearn J.M. The search for lupus biomarkers. Best. Pract. Res. Clin. Rheumatol., 23: $507-$ 23, 2009 .

16- MOTOMURA T., SHIRABE K., MANO Y., et al.: Neutrophillymphocyte ratio reflects hepatocellular carcinoma recurrence after liver transplantation via inflammatory microenvironment. J. Hepatol., 58: 58-64, 2013.

17- RAJESH B. and TAK S.: Predictive prognostic value of neutrophil-lymphocytes ratio in acute coronary syndrome. Indian Heart Journal, 69: S46-S50, 2017.

18- RANDY B.C.: Neutrophil-to-lymphocyte ratio as prognostic indicator in gastrointestinal cancers: A systematic review and meta-analysis, 8 (19): 32171, 2017.

19-QIN B., MA N., TANG Q., et al.: Neutrophil to lymphocyte ratio (NLR) and platelet to lymphocyte ratio (PLR) were useful markers in assessment of inflammatory response 
and disease activity in SLE patients. Mod. Rheumatol., 26: 372-6, 2016.

20- LI L., XIA Y., CHEN C., et al.: Neutrophil-lymphocyte ratio in systemic lupus erythematosus disease: A retrospective study. International journal of clinical and experimental medicine, 8 (7): 11026, 2015.

21- DELGADO-GARCÍA, GUILLERMO, et al.: Mean platelet volume is decreased in adults with active lupus disease. Revista Brasileira de Reumatologia (English Edition), 56 (6): 504 8, 2016.

22- YOLBAS S., YILDIRIM A., BURAK U.Z., et al.: Hematological Indices May Be Useful in the Diagnosis of Systemic Lupus Erythematosus and in Determining Disease Activity in Behçet's Disease. Medical Principles and Practice, 25 (6): 510-16, 2016.

23- AYNA A.B., ERMURAT S., COŞKUN B.N., et al.: Neutrophil to Lymphocyte Ratio and Mean Platelet Volume as Inflammatory Indicators in Systemic Lupus Erythematosus Nephritis. Archives of Rheumatology, 32 (1): 21 5, 2017.

24- OEHADIAN A., SURYADINATA H., DEWI S., et al.: The role of neutrophyl lymphocyte count ratio as an inflammatory marker in systemic lupus erythematosus. Acta. Med. Indones, 45 (3): 170-4, 2013.
25- UMARE V., PRADHAN V., NADKAR M., et al.: Effect of Proinflammatory Cytokines (IL-6, TNF- a, and IL-1ß) on Clinical Manifestations in Indian SLE Patients. Mediators Inflamm., 2014 : 385297, 2014.

26- EL-SAYED Z.H., ALI S.T. and MOHAMED A.K.: Predictive Role of Plasma Neutrophil Gelatinase-associated Lip ocalin and IL-18 in Lupus nephritis. International Journal of Advanced Research, 3: 912-22, 2015.

27- WU Y., CHEN Y., YANG X., et al.: Neutrophil-tolymphocyte ratio (NLR) and platelet-to-lymphocyte ratio (PLR) were associated with disease activity in patients with systemic lupus erythematosus. International immunopharmacology, 36: 94-9, 2016.

28- LUO Q., YE J., ZENG L., et al.: Elevated expression of TIGIT on CD3+ CD4+ T cells correlates with disease activity in systemic lupus erythematosus. Allergy, Asthma \& Clinical Immunology, 13 (1): 15, 2017.

29- YAVUZ S. and ECE A.: Mean platelet volume as an indicator of disease activity in juvenile SLE. Clinical rheumatology, 33 (5): 637-41, 2014.

30- ELWY M.A., GALAL Z.A., and HASAN H.E.: Immunoinflammatory markers and disease activity in systemic lupus erythematosus: Something old, something new. East. Mediterr. Health. J., 16 (8): 893-900, 2010.

\title{
نسبة الكرات البيضاء المتعادلة إلى الخلايا الليمفاوية كدلالة تنبؤية الئية

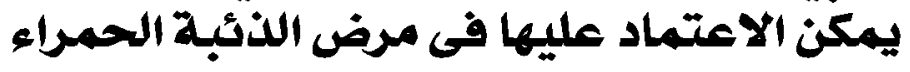

\author{
آجريت هذه الدراسة على آربعين مريضا يعانون من مرض الذئبة الحمراء النشطة وتم تقسيمها إلى مجموعتين (أ) ثالاثين مريضا يعانون

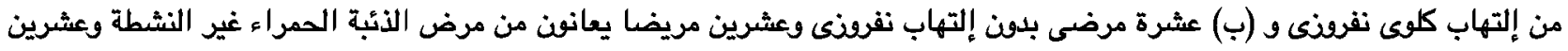

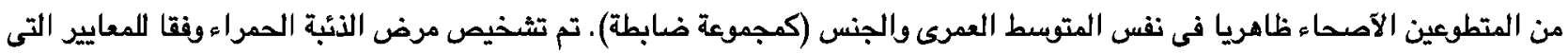

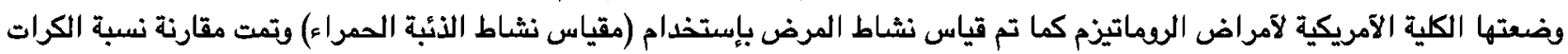

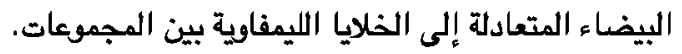 \\ وقد خلصت الدراسة إلى إمكانية إستخدام نسبة الكرات البيضاء المتعادلة إلى الخلايا الليمفاوية كدلالة لقياس نشاط مرض الذئبة الحمراء \\ والتنبؤ بإلالتهاب النفروذى .
}

